Nereis. Revista Iberoamericana Interdisciplinar de Métodos, Modelización y Simulación
12

43-54
Universidad Católica de

Valencia San Vicente Mártir
Valencia

(España)

\title{
Aplicación de la topología molecular a la predicción de la actividad antiparasitaria frente a Giardia intestinalis y Trichomonas vaginalis de derivados del 2-Acylamino-nitro-1,3-tiazol
}

\author{
Application of molecular topology to the prediction of antiparasitic activity against \\ Giardia intestinalis and Trichomonas vaginalis of 2-Acylamino-nitro-1,3-thiazole products
}

Fecha de recepción y aceptación: 24 de febrero de 2020, 2 de marzo de 2020

DOI: $10.46583 /$ nereis 2020.12 .586

\section{Izquierdo-García ${ }^{1}$, E. Lindsay-Pérez ${ }^{1}$, R. Alaoui-Bouhamid ${ }^{1}$, M. Parra-Civera ${ }^{1}, J$. Gálvez ${ }^{2}$ y R. García-Domenech ${ }^{2 *}$}

${ }^{1}$ Departamento de Farmacia y Tecnología Farmacéutica y Parasitología. Facultad de Farmacia. Universitat de València.

${ }^{2}$ Departamento de Química Física. Facultad de Farmacia. Universitat de València.

${ }^{*}$ Correspondencia: Universitat de València. Facultad de Farmacia. Departamento de Química Física. Avda. V. A. Estellés, s/n. 46100 (Burjassot), Valencia. España. E-mail: ramon.garcia@uv.es

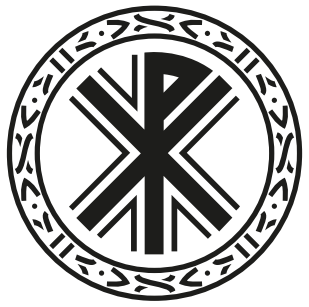

RESUMEN

Giardia intestinalis y Trichomonas vaginalis destacan por su importancia clínica. G. intestinalis causa la giardiosis, una parasitosis de gran importancia epidemiológica y clínica por presentar una elevada prevalencia. T. vaginalis causa la tricomoniasis, la enfermedad de transmisión sexual (ETS) no viral con mayor incidencia del mundo. Ambas parasitosis comparten el mismo tratamiento farmacológico: los nitroimidazoles. Se ha aplicado la topología molecular en la búsqueda de derivados del 2-Acylamino-nitro-1,3-tiazol con actividad antiparasitaria frente a G. intestinalis y T. vaginalis. Con el análisis lineal discriminante se obtuvo un modelo capaz de clasificar correctamente el $92,85 \%$ de los compuestos estudiados en ambos parásitos. Para predecir la actividad antiparasitaria, se llevó a cabo un análisis de regresión multilineal capaz de explicar el 83,2 \% de la varianza en G. intestinalis, y el 89,4 \% en T. vaginalis. Por último, se llevó a cabo un cribado molecular para buscar nuevos compuestos potencialmente activos frente a ambos parásitos.

PALABRAS CLAVE: topología molecular, QSAR, Giardia intestinalis, Trichomonas vaginalis.

\section{ABSTRACT}

Giardia intestinalis and Trichomonas vaginalis stand out for their clinical importance. G. intestinalis causes giardiasis, a parasitism of great epidemiological and clinical importance due to its high prevalence. T. vaginalis causes trichomoniasis, the non-viral sexually transmitted disease (STD) with the highest incidence in the world. Both parasitisms share the same pharmacological treatment: nitroimidazoles. Molecular topology has been applied in searching for derivatives of 2-Acylamino-nitro-1,3-thiazole with antiparasitic activity against $G$. intestinalis and $T$. vaginalis. 
After using linear discriminant analyses, results show a model that could correctly classify the activity of $92.85 \%$ of the compounds studied in both parasites. To predict antiparasitic activity, a multilinear regression analysis was carried out that could explain $83.2 \%$ of the variance in $G$. intestinalis, and $89.4 \%$ in T. vaginalis. Finally, a molecular screening was carried out to look for new potentially active compounds against both parasites.

KEYWORDS: molecular topology, QSAR, Giardia intestinalis, Trichomonas vaginalis.

\section{INTRODUCCIÓN}

Giardia intestinalis y Trichomonas vaginalis son parásitos que destacan por su importancia clínica. Tienen un ciclo biológico sencillo y el mismo tratamiento farmacológico: los nitroimidazoles.

La giardiosis es causada por el parásito G. intestinalis. Se trata de una parasitosis de gran importancia epidemiológica y clínica por su alta prevalencia, principalmente en la población infantil [1]. Suele resolverse de forma espontánea; sin embargo, existen ocasiones en las que la parasitación puede durar semanas o meses en ausencia de tratamiento. La sintomatología gastrointestinal es la más frecuente con una serie de manifestaciones clínicas entre las cuales destaca la enteritis aguda o la diarrea crónica. Los nitroimidazoles constituyen el tratamiento de elección [2,3].

La tricomoniasis es la enfermedad de transmisión sexual (ETS) no viral con mayor incidencia del mundo, existen 276 millones de casos al año [4]. Se produce a través de la transmisión del parásito protozoario T. vaginalis. Se trata de un parásito de distribución cosmopolita que se localiza habitualmente en la vagina de la mujer y en las glándulas prostáticas del varón. Predomina en mujeres en edad fértil, con un pico entre los 16 y 35 años, grupo etario en el que se estima que entre el 10 y $30 \%$ están infectadas [5]. El tratamiento recomendado es el uso de metronidazol o tinidazol por vía oral [6].

El tratamiento frente a diversas enfermedades parasitarias está limitado en numerosas ocasiones por la escasa existencia de fármacos en el mercado, la mayoría de los cuales son de baja eficacia, muestran elevados efectos secundarios y aparecen con mayor frecuencia cepas resistentes que dificultan su tratamiento y control [7]. Esto refleja la necesidad de continuar con la búsqueda constante de mejores fármacos antiprotozoarios con la finalidad de combatir estas dificultades [8].

Hoy en día, el estudio de la relación cuantitativa estructura-actividad "Quantitative Structure Activity Relationship" (QSAR) se considera una herramienta tan valiosa como básica en el campo de la investigación sobre desarrollo de nuevos fármacos. Se integra dentro de las técnicas in silico, también llamadas técnicas por simulación computacional $[8,9]$.

La interrelación de la informática, la biología, la química y las matemáticas está haciendo posible realizar experimentación virtual que presenta varias ventajas, entre las cuales destacan la rapidez, la reducción de costes, el manejo de un número muy elevado de moléculas y la disminución del uso de animales de experimentación. En este ámbito encuentra su utilidad la topología molecular, ya que permite encontrar relaciones entre propiedades biológicas y/o físico-químicas y estructuras moleculares [10]. Mediante el uso de índices o descriptores topológicos, es posible predecir de manera precisa la actividad farmacológica de un compuesto [8,9].

La topología molecular ha sido aplicada con éxito en el campo de los antimaláricos $[11,12,13]$ y en la lucha contra los vectores transmisores de enfermedades parasitarias $[14,15]$. 
El trabajo presentado tiene como objetivo aplicar la topología molecular mediante el análisis QSAR para predecir la actividad antiparasitaria de un grupo de compuestos derivados del 2-acylamino-nitro-1,3-tiazol frente a los protozoos G. intestinalis y T. vaginalis. Para ello se utiliza el análisis lineal discriminante, ALD, y el análisis de regresión multilineal, ARML. Una vez validados los modelos topológicos de predicción, se realiza un cribado molecular para la búsqueda de nuevos compuestos potencialmente activos frente a ambos parásitos.

\section{MATERIAL Y MÉTODOS}

\section{Compuestos analizados}

La topología molecular requiere la obtención de bibliotecas de datos para poder trabajar [9]. En este estudio han sido seleccionados 15 compuestos (tabla 1), cuyos datos de actividad se obtuvieron

Tabla 1. Estructura química y actividad antiparasitaria, $\mathrm{IC}_{50}(\mu \mathrm{M})$, frente a G. intestinalis y T. vaginalis de los compuestos analizados, derivados del 2-acylamino-5-nitrotiazol [7]

\begin{tabular}{|c|c|c|c|c|c|}
\hline \multirow[b]{2}{*}{ Compuesto } & \multirow[b]{2}{*}{ Z } & \multicolumn{2}{|c|}{ G. intestinalis } & \multicolumn{2}{|c|}{ T. vaginalis } \\
\hline & & $\mathrm{IC}_{50}$ & $\mathrm{pIC}_{50}$ & $\mathrm{IC}_{50}$ & $\mathrm{pIC}_{50}$ \\
\hline 1 & Cyclohexylamine & 0,359 & 6,44 & 1,650 & 5,78 \\
\hline 2 & Phenylamine & 2,570 & 5,59 & - & \\
\hline 3 & 4-Chlorophenylamine & 0,577 & 6,23 & 1,026 & 5,99 \\
\hline 4 & 4-Fluorphenylamine & 7,826 & 5,10 & 1,226 & 5,91 \\
\hline 5 & 4-Methoxyphenylamine & 29,11 & 4,53 & 1,300 & 5,89 \\
\hline 6 & 4-Ehtoxyphenylamine & 3,747 & 5,42 & 0,282 & 6,55 \\
\hline 7 & 4-Butoxyphenylamine & 1,800 & 5,74 & 0,387 & 6,41 \\
\hline 8 & 4-Nitrophenylamine & 15,34 & 4,81 & 0,958 & 6,02 \\
\hline 9 & Methyl & 0,490 & 6,30 & 0,022 & 7,66 \\
\hline 10 & Phenyl & 0,481 & 6,31 & 0,124 & 6,91 \\
\hline 11 & Butyl & 0,398 & 6,40 & 0,305 & 6,52 \\
\hline 12 & Pentadecyl & 1,634 & 5,78 & 2,584 & 5,59 \\
\hline 13 & Methoxy & 0,010 & 8,00 & 0,081 & 7,09 \\
\hline 14 & Ethoxycarbonyl & 6,410 & 5,19 & 0,010 & 8,00 \\
\hline Nitazoxanida & 2-Acetoxyphenyl & 1,214 & 5,91 & 0,068 & 7,17 \\
\hline
\end{tabular}


del estudio realizado por Nava-Zuazo et al. [7]. Estos compuestos se diseñaron sobre la estructura del fármaco antiprotozoario nitazoxanida, manteniendo la estructura del 5-nitrotiazol y añadiendo en la posición (Z) los grupos funcionales que hay que examinar. Todas las moléculas se dibujaron en 2D mediante el programa informático ChemDraw ${ }^{\circledR}$ Professional versión 16.0 [16].

Los nuevos compuestos se nombraron del 1 al 14 más el fármaco de referencia, nitazoxanida, manteniendo la numeración original del estudio de Nava-Zuazo et al. [7]. Fueron dibujados y guardados en archivos .mol desde el programa ChemDraw ${ }^{\circledR}$ Professional versión 16.0, para su posterior utilización para el cálculo de los índices topológicos (IT) de cada molécula.

\section{Descriptores moleculares}

La singularidad de los modelos QSAR desarrollados consiste en la exclusiva utilización de descriptores topológicos. Los IT codifican información acerca de la estructura molecular de manera numérica. Estos formatos numéricos simplifican la búsqueda de otras moléculas con propiedades muy similares, permitiendo la selección o el diseño de nuevos fármacos [9]. Cada compuesto está caracterizado por una serie específica de descriptores topológicos.

En este trabajo, los IT utilizados para determinar la actividad de las moléculas diseñadas fueron diversos. Una ventaja sustancial del empleo de descriptores topológicos es que se pueden emplear en un sentido inverso, bien partiendo del IT a la propiedad molecular o de la propiedad molecular al IT. Los índices topológicos se obtuvieron utilizando el programa de cálculo DRAGON® versión 5.0. [17]. Este software informático permite el cálculo de un gran número de IT.

\section{Algoritmos QSAR}

\section{Análisis lineal discriminante}

El objetivo del análisis lineal discriminante (ALD) es determinar una función lineal capaz de discriminar entre compuestos activos o inactivos según los valores de los diferentes descriptores moleculares [9]. En este trabajo, los IT se utilizan para generar el modelo topológico.

Mediante el ALD también se puede determinar la especificidad y la sensibilidad de la función discriminante, FD. La sensibilidad se refiere a la capacidad de detectar compuestos activos y la especificidad como la capacidad de detectar compuestos inactivos.

Cada compuesto se clasificó como activo o inactivo en base a su capacidad antiprotozoaria. En el caso de $G$. intestinalis, el fármaco de referencia fue la nitazoxanida $\left(\mathrm{IC}_{50}=1,214 \mu \mathrm{M}, \mathrm{pIC}_{50}=5,91\right)$. Las moléculas se consideraron activas $\mathrm{si}_{\mathrm{pIC}}>5,91$, clasificándolas dentro del grupo A; mientras que todas aquellas moléculas con valores inferiores se clasificaron en el grupo I. En el caso de T. vaginalis, el fármaco de referencia fue el metronidazol $\left(\mathrm{IC}_{50}=0,29 \mu \mathrm{M}, \mathrm{pIC}_{50}=6,54\right)$. Las moléculas se consideraron activas si $\mathrm{pIC}_{50}>6,54$, clasificándolas dentro del grupo A; mientras que aquellas moléculas con valores inferiores se clasificaron en el grupo I. 
El software utilizado fue el Statistica ${ }^{\circledR}$ versión 8.0. [18]. La capacidad discriminante se evaluó a través del porcentaje de clasificación correcta para cada grupo. El criterio de clasificación se basa en la distancia mínima de Mahalanobis (la distancia de cada caso a la media de todos los casos en una categoría). La calidad de discriminación se evaluó utilizando la lambda de Wilks $(\lambda)$, indicando que cuanto más pequeño sea el valor de este parámetro, $\lambda$, menor será la superposición entre el grupo activo y el inactivo, obteniendo así una mejor separación de los grupos.

Una vez obtenida la función discriminante (FD) se realizó un diagrama de distribución de la actividad antiprotozoaria para ambos parásitos. Estos diagramas son histogramas en los cuales el eje de ordenadas representa la expectancia (E) y el eje de abscisas el valor de la FD. Permiten observar los intervalos de la FD, de forma que se puede visualizar si la probabilidad de actividad es máxima o mínima, y por tanto en qué intervalos hay mayor probabilidad de encontrar compuestos activos [19]. Es una manera de encontrar el dominio de aplicabilidad de la FD.

\section{Análisis de regresión multilineal}

El objetivo general del ARML es definir la relación entre dos o más variables independientes y una variable dependiente proporcionando una ecuación lineal para la predicción de la propiedad seleccionada.

El ARML es un algoritmo lineal que supone una combinación lineal de descriptores adecuados para cuantificar la actividad antiprotozoaria de un compuesto $\left(\mathrm{plC}_{50}\right)$. En este estudio, se obtuvo toda la información necesaria utilizando el software de Statistica ${ }^{\circledR}$ versión 8.0 [18].

Las etapas que hay que seguir en el ARML fueron las siguientes: en primer lugar, una selección de la mejor ecuación de predicción basada en la transformación de variables, el método que hay que seguir y el criterio estadístico para la selección de variables, el valor del coeficiente de correlación múltiple $\left(\mathrm{R}^{2}\right)$, el número de variables en la ecuación, la significación estadística $(\mathrm{p}<0,005)$, el error estándar de estimación (EEE) ( $<10 \%$ del intervalo de la propiedad estudiada) y la intercorrelación entre variables. Y en segundo lugar, el test de validación interna, que puede hacerse mediante crosvalidación tipo leave-one-out o mediante la crosvalidación tipo leave-n-out. En este estudio se realizó la crosvalidación tipo leave-one-out. Para ello, se elimina un compuesto de la data y se obtiene la función de regresión con el resto de los compuestos, usando los mismos IT, y se calcula el valor de la propiedad del compuesto eliminado. El proceso se repite tantas veces como compuestos hay en la data. Con los datos de predicción obtenidos, se calcula el coeficiente de predicción $\mathrm{Q}^{2}$.

\section{RESULTADOS Y DISCUSIÓN}

En primer lugar, se llevó a cabo el ALD para el parásito G. intestinalis, utilizando la variable de agrupación Clas. (A, I) como variable dependiente y los IT como variables independientes.

La FD seleccionada fue la siguiente:

$$
\begin{aligned}
& \mathrm{FD}_{\text {Gint }}=147,3-42,6 \times \mathrm{piPC} 02+1678,1 \times \mathrm{JGI} 10 \\
& \mathrm{~N}=15 \quad \lambda=0,27 \quad \mathrm{~F}(2,12)=16,35 \quad \mathrm{p}<0,0004
\end{aligned}
$$

Ec. 1 
Los índices topológicos que aparecen en la ecuación 1 (Ec.1) son: piPC02, número de caminos múltiples de orden 2 [20]; y JGI10, índice topológico de carga ponderado por enlace de orden 10 [21].

Se obtuvo una alta significación estadística $(\mathrm{p}<0,0004)$ y un coeficiente $\lambda$ de Wilk's $(0,27)$ que sugiere un elevado grado de discriminación.

La FD clasifica a los compuestos como activos (A) si FD $>0$ o inactivos (I) si FD $<0$.

De los 15 compuestos analizados, 7 forman el grupo activo y 8 el inactivo. Como se observa en la tabla 2, la FD ha clasificado como activos a 6 de 7 moléculas del grupo A, mientras que ha clasificado como inactivos a 8 de las 8 moléculas del grupo I. Ello implica una sensibilidad del 100,0\%, una especificidad del $88,9 \%$ y una exactitud del 93,3\% de clasificación correcta del grupo de compuestos estudiado.

Tabla 2. Resultados del ALD y el ARML para cada compuesto estudiado en G. intestinalis

\begin{tabular}{|c|c|c|c|c|c|c|c|}
\hline Compuesto & $\mathrm{plC}_{50}$ Exp. $^{\mathrm{a}}$ & $\begin{array}{c}\text { Clasif. } \\
\text { Exp. }\end{array}$ & Prob.(A) ${ }^{b}$ & $\mathrm{FD}^{\mathrm{c}}$ & $\begin{array}{c}\text { Clasif. } \\
\text { Calc. }\end{array}$ & pIC50 Calc. ${ }^{\mathrm{d}}$ & $\begin{array}{c}\text { pIC50 } \\
\text { Calc. (vc) }\end{array}$ \\
\hline 1 & 6,44 & A & 0,998 & 6,37 & A & 6,42 & 6,41 \\
\hline 2 & 5,59 & I & 0,205 & $-1,17$ & I & 5,88 & 5,90 \\
\hline 3 & 6,23 & A & 0,290 & $-0,71$ & I & 6,02 & 5,96 \\
\hline 4 & 5,10 & I & 0,290 & $-0,71$ & I & 5,05 & 5,04 \\
\hline 5 & 4,53 & I & 0,006 & $-4,96$ & I & 5,33 & 5,44 \\
\hline 6 & 5,42 & I & 0,002 & $-5,86$ & I & 5,59 & 5,62 \\
\hline 7 & 5,74 & I & 0,000 & $-9,28$ & I & 5,49 & 5,46 \\
\hline 8 & 4,81 & I & 0,000 & $-8,46$ & I & 4,96 & 4,99 \\
\hline 9 & 6,30 & A & 0,999 & 6,89 & A & 6,54 & 6,74 \\
\hline 10 & 6,31 & A & 1,000 & 8,20 & A & 5,67 & 5,60 \\
\hline 11 & 6,40 & A & 0,905 & 2,42 & A & 6,00 & 5,92 \\
\hline 12 & 5,78 & I & 0,012 & $-4,20$ & I & 5,64 & 5,63 \\
\hline 13 & 8,00 & A & 0,994 & 5,36 & A & 8,13 & 8,44 \\
\hline 14 & 5,19 & I & 0,044 & $-2,91$ & I & 5,40 & 5,46 \\
\hline Nitazoxanida & 5,91 & A & 0,992 & 4,97 & A & 5,63 & 5,59 \\
\hline
\end{tabular}

${ }^{a}$ Valores pIC ${ }_{50}$ Exp. $(\mu \mathrm{M})$ obtenidos a partir de Nava-Zuazo et al. [7]

${ }^{b}$ Probabilidad de pertenecer al grupo activo

${ }^{\mathrm{c}}$ Valores obtenidos mediante Ec. 1

${ }^{\mathrm{d}}$ Valores obtenidos mediante Ec. 3

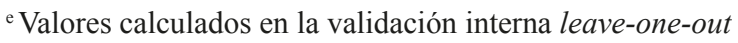

A: compuesto clasificado como activo; I: compuesto clasificado como inactivo

A continuación se llevó a cabo el mismo procedimiento para el parásito T. vaginalis.

La FD seleccionada fue la siguiente:

$\mathrm{FD}_{\text {Tvag }}=-354,6+124,1 \times$ ATS5e $-73,1 \times$ EEig11d

Ec. 2

$$
\mathrm{N}=15 \quad \lambda=0,19 \quad \mathrm{~F}(2,12)=23,9 \quad \mathrm{p}<0,0001
$$


Los índices topológicos que intervienen en la Ec 2 son: ATS5e, índice de autocorrelación de BrotoMoreau de orden 5 ponderado por las electronegatividades atómicas de Sanderson y EEig11d, autovalor de orden 11 obtenido de la matriz de adyacencia de aristas ponderada por los momentos dipolares.

Se obtuvo una alta significación estadística $(\mathrm{p}<0,0001)$ y un coeficiente $\lambda$ de Wilk's $(0,19)$ que sugiere un elevado grado de discriminación.

El grupo de compuestos analizados con este parásito fue de 14, de ellos 7 formaban el grupo activo y otros 7 el grupo inactivo. Como se observa en la tabla 3, la FD ha clasificado como activos a 6 de 7 moléculas del grupo A, mientras que ha clasificado como inactivos a 7 de las 7 moléculas del grupo I. Por tanto, la matriz de clasificación muestra una sensibilidad del 100,0 \%, una especificidad del $87,5 \%$ y un porcentaje de clasificación correcta del $92,9 \%$.

La tabla 3 muestra la actividad de los 15 compuestos estudiados, la FD y la actividad predicha de cada uno tras aplicar el ALD y el ARML.

Tabla 3. Resultados del ALD y el ARML para cada compuesto estudiado en T. vaginalis

\begin{tabular}{cccccccc}
\hline Compuesto & $\mathrm{pIC}_{50}$ Exp. $^{\mathrm{a}}$ & Clasif. Exp. & Prob.(A) & FD. & Clasif. Calc. $^{\mathrm{b}}$ & $\mathrm{pIC}_{50}$ Calc. $^{\mathrm{d}}$ & $\begin{array}{c}\mathrm{pIC}_{50} \\
\text { Calc.(vc) }^{\mathrm{e}}\end{array}$ \\
\hline 1 & 5,78 & $\mathrm{I}$ & 0,000 & $-9,25$ & $\mathrm{I}$ & 5,77 & 5,78 \\
2 & - & - & - & - & - & - & - \\
3 & 5,99 & $\mathrm{I}$ & 0,000 & $-7,56$ & $\mathrm{I}$ & 6,13 & 6,16 \\
4 & 5,91 & $\mathrm{I}$ & 0,000 & $-5,66$ & $\mathrm{I}$ & 6,05 & 6,06 \\
5 & 5,89 & $\mathrm{I}$ & 0,000 & $-9,72$ & $\mathrm{I}$ & 5,91 & 5,92 \\
6 & 6,55 & $\mathrm{~A}$ & 0,200 & $-1,39$ & $\mathrm{I}$ & 6,05 & 6,00 \\
7 & 6,41 & $\mathrm{I}$ & 0,000 & $-5,52$ & $\mathrm{I}$ & 6,24 & 6,22 \\
8 & 6,02 & $\mathrm{I}$ & 0,040 & $-3,12$ & $\mathrm{I}$ & 6,15 & 6,17 \\
9 & 7,66 & $\mathrm{~A}$ & 1,000 & 10,64 & $\mathrm{~A}$ & 7,35 & 6,99 \\
10 & 6,91 & $\mathrm{~A}$ & 0,990 & 4,28 & $\mathrm{~A}$ & 6,78 & 6,77 \\
11 & 6,52 & $\mathrm{~A}$ & 1,000 & 10,72 & $\mathrm{~A}$ & 6,35 & 6,32 \\
12 & 5,59 & $\mathrm{I}$ & 0,000 & $-11,60$ & $\mathrm{I}$ & 5,53 & 5,54 \\
13 & 7,09 & A & 1,000 & 8,78 & $\mathrm{~A}$ & 7,53 & 7,74 \\
14 & 8,00 & A & 1,000 & 7,55 & $\mathrm{~A}$ & 7,79 & 7,64 \\
Nitazoxanida & 7,17 & A & 1,000 & 11,60 & A & 7,19 & 7,26 \\
\hline
\end{tabular}

a Valores pIC50 Exp. ( $\mu \mathrm{M})$ obtenidos a partir de Nava-Zuazo et al. [7]

${ }^{\mathrm{b}}$ Probabilidad de pertenecer al grupo activo

${ }^{\mathrm{c}}$ Valores obtenidos mediante Ec. 2

'Valores obtenidos mediante Ec. 4

e Valores calculados en la validación interna leave-one-out

A: compuesto clasificado como activo; I: compuesto clasificado como inactivo

A partir de los resultados y tras aplicar la FD a cada compuesto se realizaron los diagramas de distribución de la actividad para ambos parásitos (figura 1). Las bandas blancas representan al grupo inactivo, mientras que las negras al grupo activo. Los diagramas de actividad muestran una capacidad discriminante muy eficiente para ambos parásitos. En el caso de G. intestinalis (figura 1A) se observa 
que la mayor probabilidad de encontrar compuestos activos se da para intervalos de $\mathrm{FD}_{\text {Gint }}$ entre 0 y 10 , mientras que para $T$. vaginalis (figura B), el intervalo se sitúa entre 0 y 12 . Estos intervalos se eligieron como dominio de aplicación de ambas FD.
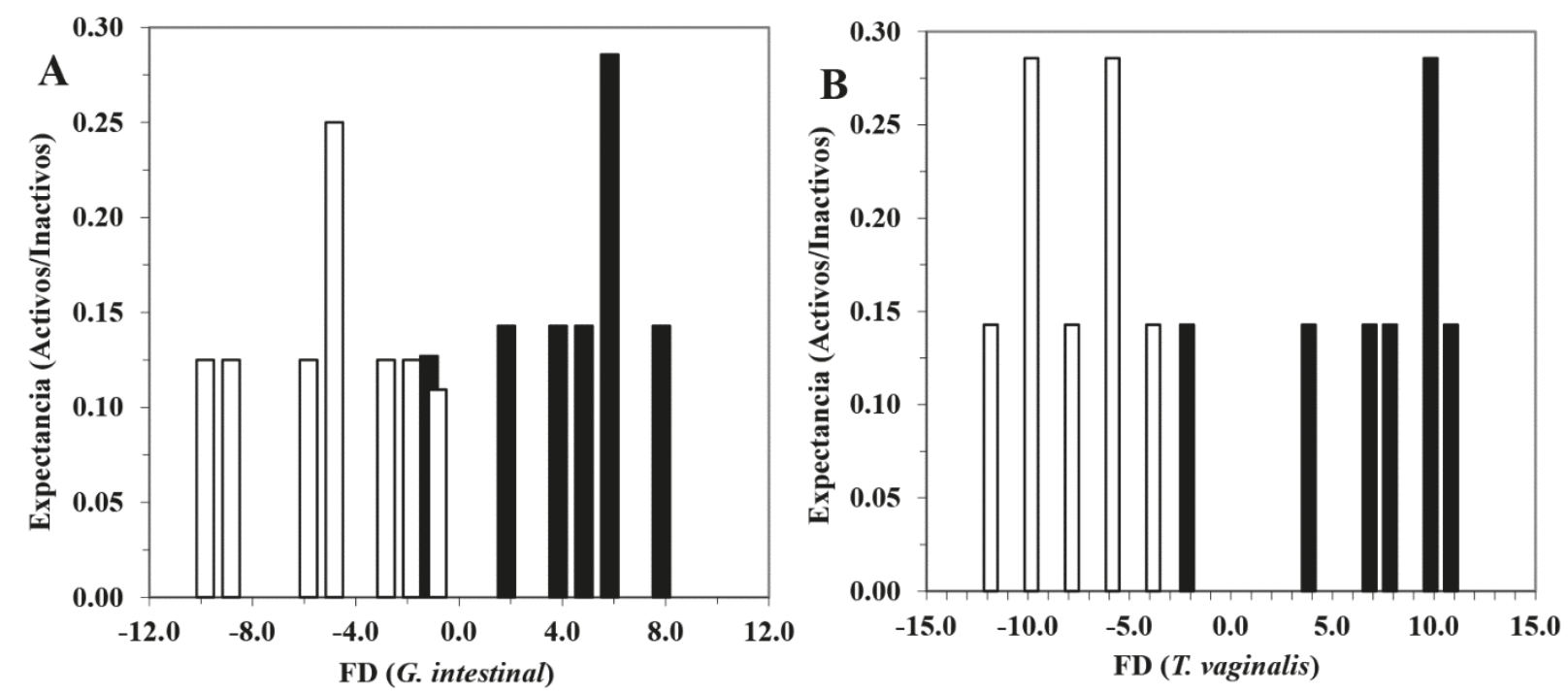

Fig. 1. Diagramas de distribución de la actividad antiprotozoaria frente a G. intestinalis (fig. A) y T. vaginalis (fig. B), obtenidos con las funciones $\mathrm{FD}_{\text {Gint } \mathrm{y}} \mathrm{FD}_{\mathrm{Tvag}}$.

El ARML se desarrolló para obtener funciones de predicción de la actividad antiparasitaria para cada parásito.

En primer lugar, se aplicó para $G$. intestinalis, utilizando la variable $\mathrm{plC}_{50}$ como variable dependiente y los índices topológicos como variables independientes.

A partir de los datos se seleccionó la siguiente ecuación de predicción:

$$
\begin{aligned}
& \mathrm{pIC} 50_{\text {Gint }}=18,33-7,49 \times \text { GATS4m - 2,21 x EEig05x } \\
& \mathrm{N}=15 \quad \mathrm{R}^{2}=0,832 \quad \mathrm{Q}^{2}=0,768 \quad \mathrm{SEE}=0,373 \quad \mathrm{~F}(2,12)=29,7 \quad \mathrm{p}<0,00002
\end{aligned}
$$

Se observa una alta significación estadística $(\mathrm{p}<0,00002)$, una correlación aceptable $\left(\mathrm{R}^{2}=0,832\right)$ y un error de estándar de estimación $(\mathrm{EEE}=0,373)$ que equivale al 10,7 \% de la variabilidad en la que se mueve la propiedad estudiada (valor de la incertidumbre que acompaña a la predicción de la propiedad). La crosvalidación muestra un coeficiente de predicción de $\mathrm{Q}^{2}=0,768$, por tanto, la función obtenida se considera predictiva.

Los índices topológicos expresados en la Ec.3 son: GATS4m, índice de autocorrelación de Geary de orden 4 ponderado por masas atómicas y EEig05x, un autovalor de orden 5 obtenido a partir de la matriz de adyacencia de aristas ponderada por orden de enlace. 
Los valores de $\mathrm{pIC}_{50}$ calculados y obtenidos mediante la Ec.3 se muestran en la tabla 2, en la cual se observa que comparten similitud con los valores experimentales ( $\mathrm{pIC}_{50}$ Exp.) de G. intestinalis.

A continuación, se llevó a cabo el mismo procedimiento para el parásito T. vaginalis, obteniendo la siguiente ecuación de predicción:

$$
\begin{aligned}
& \text { plC50 } 0_{\text {Tvag }}=-2,86+4,44 \times \text { ATS5m - 2,29 x ATS8p Ec. } 4 \\
& \mathrm{~N}=14 \quad \mathrm{R}^{2}=0,894 \quad \mathrm{Q}^{2}=0,790 \quad \mathrm{SEE}=0,265 \quad \mathrm{~F}(2,11)=46,59 \quad \mathrm{p}<0,000004
\end{aligned}
$$

Se observa una alta significación estadística $(p<0,000004)$, una correlación adecuada $\left(R^{2}=0,894\right)$ y un error de estándar de estimación ( $\mathrm{EEE}=0,265$ ) que equivale al $10,6 \%$ de la variabilidad en la que se mueve la propiedad estudiada. La validación cruzada muestra un coeficiente de predicción (tipo leave-one-out) de $\mathrm{Q}^{2}=0,790$, por tanto, la función obtenida se considera predictiva.

Los índices topológicos que aparecen en la Ec.4 corresponden a índices de autocorrelación de Broto-Moreau que evalúan la similaridad topológica existente entre las moléculas estudiadas.

Los valores de $\mathrm{pIC}_{50}$ predichos con la Ec.4 para cada compuesto se muestran en la tabla 3. Como puede observarse, existe una concordancia muy aceptable entre los valores experimentales y los calculados $\left(\mathrm{pIC}_{50 \text { Exp }}, \mathrm{pIC}_{50 \text { Calc. }}\right.$ ) de T. vaginalis.

Tras haber obtenido un modelo topológico-matemático de predicción con buenos resultados de la actividad antiprotozoaria para cada compuesto y parásito analizado, se procedió a realizar un cribado molecular. Se propusieron 40 nuevas moléculas derivadas del 2-Acylamino-nitro-1,3-tiazol usando la estructura base mostrada en la tabla 1, con el fin de buscar nuevos compuestos potencialmente activos para ambos parásitos. A cada una de las moléculas diseñadas se les aplicaron las funciones $\mathrm{FD}_{\text {Gint, }}$, $\mathrm{FD}_{\text {Tvag }}$ pIC50 $0_{\text {Gint }}$ y pIC50 Tvag.

De todas las moléculas propuestas, cinco de ellas mostraron buenos resultados en cuanto a actividad antiprotozoaria para ambos parásitos y resultaron ser potencialmente activas. Estas moléculas se muestran en la figura 2 junto con los valores de $\mathrm{IC}_{50}$ y $\mathrm{FD}$.

En el caso de G. intestinalis las cinco moléculas han demostrado ser potencialmente más activas que la nitazoxanida $\left(\mathrm{IC}_{50}=1,214 \mu \mathrm{M}\right)$ y el metronidazol $\left(\mathrm{IC}_{50}=5,360 \mu \mathrm{M}\right)$, mientras que para $T$. $v a$ ginalis también se consideran potencialmente más activas (excepto el compuesto E) en comparación con los fármacos de referencia. 


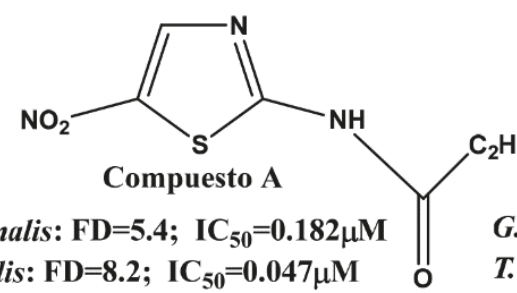

G. intestinalis: $\mathrm{FD}=3.9 ; \mathrm{IC}_{50}=0.501 \mu \mathrm{M}$

G. intestinalis: $\mathrm{FD}=5.4 ; \mathrm{IC}_{50}=0.182 \mu \mathrm{M}$

T. vaginalis: $\mathrm{FD}=8.2 ; \mathrm{IC}_{50}=0.047 \mu \mathrm{M}$

T. vaginalis: $\mathrm{FD}=3.2 ; \mathrm{IC}_{50}=0.014 \mu \mathrm{M}$ ○

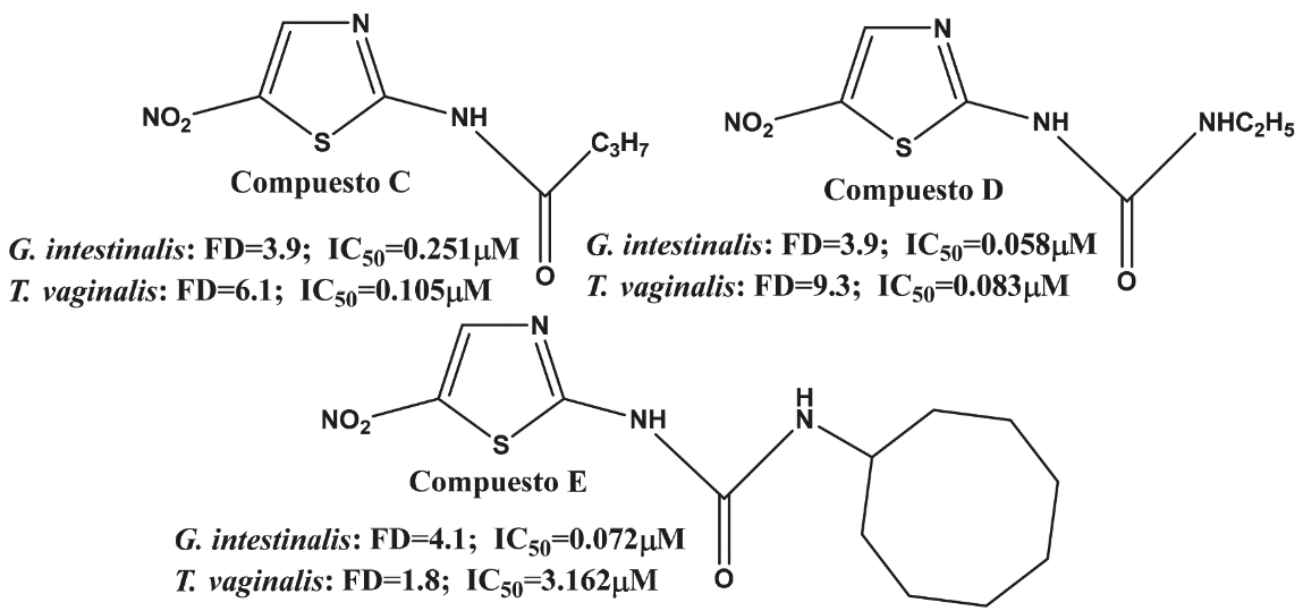

Fig. 2. Estructura de las nuevas moléculas potencialmente activas con sus valores de $\mathrm{IC}_{50} \mathrm{y}$ FD.

\section{CONCLUSIONES}

Tras analizar la actividad de los 15 compuestos derivados del 2-Acylamino-nitro-1,3-tiazol frente a $G$. intestinalis y $T$. vaginalis, se han obtenido excelentes resultados de predicción, tanto con el análisis lineal discriminante a través de las funciones FD para cada parásito, con una sensibilidad del $100 \%$ y una especificidad superior al $85 \%$, como con el análisis de regresión multilineal, para predecir la actividad $\mathrm{pIC}_{50}$ para cada parásito. Por tanto, la topología molecular ha demostrado ser una herramienta eficaz para la búsqueda de modelos QSAR en la predicción de la actividad antiparasitaria frente a ambos parásitos. Además, el modelo obtenido puede ser aplicado mediante cribado molecular para la búsqueda de nuevos compuestos potencialmente activos frente a G.intestinalis y T.vaginalis.

\section{AGRADECIMIENTOS}

Agradecemos al Máster en Enfermedades Parasitarias Tropicales de la Universitat de València por haber permitido realizar este trabajo. JG y RGD agradecen al Ministerio de Economía, Industria y Competitividad de España (Proyecto "Desarrollo de nuevas herramientas para el control de oidios", AGL2016-76216-C2-2-R) el apoyo recibido para la realización del trabajo. 


\section{REFERENCIAS BIBLIOGRÁFICAS}

[1] Centers for Disease Control and Prevention. Giardiasis. U.S. Department of Health \& Human Services. 2017. [Consultado: 16 de febrero de 2020]. Disponible en: https://www.cdc.gov/parasites/giardia/index.html

[2] Hugo DL. Giardia y giardiasis. Instituto de Investigaciones Médicas Mercedes y Martín Ferreyra. 2006;66:70-4.

[3] Michael JG. Giardiasis. Parasitic diseases of the liver and intestines. 1996;25:493-515.

[4] Alexandra IE, Alicia GB. Trichomonas vaginalis: la versatilidad de un parásito tenaz. Real Acad Nac Farm. 2017;83(1):10-47.

[5] Heine P, McGregor JA. Trichomonas vaginalis: a reemerging pathogen. Clin Obstet Gynecol. 1993;36:137-44.

[6] Centers for Disease Control and Prevention. Trichomoniasis. U.S. Department of Health \& Human Services. 2017. [Consultado: 16 de febrero de 2020]. Disponible en: https://www.cdc.gov/ std/trichomonas/default.htm

[7] Nava-Zuazo C, Chávez-Silva F, Moo-Puc R, Chan-Bacab MJ, Ortega-Morales BO, Moreno-Díaz $\mathrm{H}$ et al. 2-acylamino-5-nitro-1, 3-thiazoles: preparation and in vitro bioevaluation against four neglected protozoan parasites. Bioor Med Chem. 2014;22(5):1626-33.

[8] Gálvez J, Gálvez-Llompart R, García-Domenech R. Molecular topology as a novel approach for drug discovery. Expert Opin Drug Discov. 2012;7(2):133-53.

[9] Zanni R, Galvez-Llompart M, García-Domenech R, J. Gálvez. Latest advances in molecular topology applications for drug discovery. Expert Opin Drug Discov. 2015;10(9):945-57.

[10] Rodríguez MÁM, López-Peñalver RJS, Tomás GA, Álvarez JG, Gálvez-Llompart M, GarcíaDomenech R. Aplicación de la topología molecular a la predicción y optimización de la actividad repelente de mosquitos de derivados de la $\mathrm{N}$-acylpiperidina. Nereis. Interdisciplinary IberoAmerican Journal of Methods, Modelling and Simulation. 2014;6:19-26.

[11] Mahmoudi N, García-Domenech R, Gálvez J, Farhati K, Franetich JF, Sauerwein R et al. New active drugs against liver stages of Plasmodium predicted by molecular topology. Antimicrob Agents Chemother 2008 Apr. 52(4):1215-20.

[12] Baptista-Peraza I, Otero-Pérez C, González-Apráez S, Pertegás-Sevilla A, Galvez J, GarciaDomenech R. Aplicación de la Topología Molecular al Análisis de la Actividad Antimalárica de 4-Aminobiciclo [2.2. 2] Octan-2-il 4-Aminobutanoatos y sus Análogos Etanoatos y Propanoatos. Nereis. Interdisciplinary Ibero-American Journal of Methods, Modelling and Simullation. 2019;11:51-64.

[13] Carrillo JS, Rizza C, Álvarez BE, Hernández D, Gálvez J, García-Domenech R. Aplicación de la Topología Molecular en la búsqueda de nuevos compuestos basados en Azaauronas derivados de las Auronas naturales como potenciales antimaláricos. Nereis. Interdisciplinary Ibero-American Journal of Methods, Modelling and Simulation. 2017;9:49-62.

[14] García-Domenech R, Zanni R, Galvez-Llompart M, Galvez J, Computational design of chemical for the control of mosquitoes and their diseases (2018) CRC Press Taylor \& Francis Group, Chapter 4, 107-37. 
[15] Galvez J, de Julian-Ortiz J, Garcia-Domenech R, Application of molecular topology to the prediction of potency and selection of novel insecticides active against malaria vectors. J. Molec. Struct. Theochem. 2005;727:107-13.

[16] Perkin Elmer Informatics I. ChemDraw ${ }^{\circledR}$ Professional version 16.0.

[17] Dragon for Windows (Software for Molecular Descriptor Calculations) versión 5.0. Talete SRL. Milan, Italy, 2004.

[18] StatSoft, Inc. Statistica (Data Analysis Software System) versión 8. StatSoft. Tulsa, Ok, USA, 2008.

[19] Gálvez J, García-Domenech R, De Gregorio Alapont C, De Julián-Ortiz J, Popa L. Pharmacological distribution diagrams: a tool for de novo drug design. J Mol Graph. 1996;14(5):272-6.

[20] Todeschini R, Consonni V, Mauri, Pavan M. Dragon for windows (software for molecular descriptor calculations), version 5.4. Talete SRL. Milan, Italy 2006.

[21] Galvez J, García-Domenech R, Salabert M, Soler R. Charge indexes. New topological descriptors. J. Chem. Inf. Comput. Sci. 1994;34:520-5. 